\section{(6) OPEN ACCESS}

\title{
Increased skeletal muscle-specific microRNA in the blood of patients with COPD
}

\author{
Anna Donaldson, ${ }^{1,2}$ Samantha A Natanek, ${ }^{1,2}$ Amy Lewis, ${ }^{1}$ William D-C Man, ${ }^{2}$ \\ Nicholas S Hopkinson, ${ }^{2}$ Michael I Polkey, ${ }^{2}$ Paul R Kemp ${ }^{1}$
}

\begin{abstract}
- Additional material is published online only. To view please visit the journal online (http://dx.doi.org/10.1136/ thoraxjnl-2012-203129)

${ }^{1}$ Section of Molecular Medicine, National Heart and Lung Institute, Imperial College London, London, UK ${ }^{2}$ NIHR Respiratory Biomedical Unit, Royal Brompton Hospital and National Heart \& Lung Institute, London, UK
\end{abstract}

\section{Correspondence to} Dr Paul Kemp, Section of Molecular Medicine, National Heart and Lung Institute, Imperial College London, Sir Alexander Fleming Building, South Kensington Campus, London SW7 2AZ, UK; p.kemp@imperial.ac.uk

Received 12 December 2012 Revised 3 June 2013 Accepted 6 June 2013 Published Online First 28 June 2013

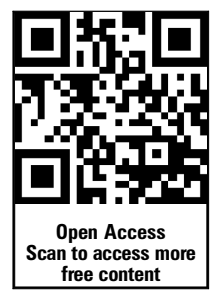

To cite: Donaldson $A$, Natanek SA, Lewis A, et al. Thorax 2013;68:

1140-1149.

\section{ABSTRACT}

Background Skeletal muscle weakness in chronic obstructive pulmonary disease (COPD) carries a poor prognosis, therefore a non-invasive marker of this process could be useful. Reduced expression of musclespecific microRNA (myomiRs) in quadriceps muscle in patients with COPD is associated with skeletal muscle weakness and changes in muscle fibre composition. Circulating exosomal miRNAs can be measured in blood, making them candidate biomarkers of biopsy phenotype. To determine whether plasma myomiR levels were associated with fibre size or fibre proportion, we measured myomiRs in plasma from patients with COPD and healthy controls.

Methods and results 103 patients with COPD and 25 age-matched controls were studied. Muscle-specific miRNA was elevated in the plasma of patients with COPD and showed distinct patterns. Specifically, miR-1 was inversely associated with fat-free mass in the cohort, whereas levels of miR-499 were more directly associated with strength and quadriceps type I fibre proportion. Two miRs not restricted to muscle in origin (miR-16 and miR-122) did not differ between patients and controls. Plasma miR-499 was also associated with muscle nuclear factor $\kappa B$ p50 but not p65 in patients with early COPD whereas plasma inflammatory cytokines were associated with miR-206 in patients with more advanced disease.

Conclusions Plasma levels of individual myomiRs are altered in patients with COPD but alone do not predict muscle fibre size or proportion. Our findings are consistent with an increase in muscle wasting and turnover associated with the development of skeletal muscle dysfunction and fibre-type shift in patients with stable COPD.

\section{INTRODUCTION}

Patients with chronic obstructive pulmonary disease (COPD) can demonstrate weakness due to reduced muscle mass and fibre size ${ }^{12}$ and reduced muscle endurance due to a shift to reduced type I muscle fibre proportions. ${ }^{3} 4$ Quadriceps weakness ${ }^{5} 6$ and wasting ${ }^{7}$ are present in spirometrically mild disease, but are predictors of mortality among patients with moderate to severe COPD, independent of lung function. ${ }^{8}$ The fibre-type shift towards a predominance of fast fibres (mainly type IIa) ${ }^{4} 9{ }^{10}$ is independently associated with exercise intolerance in COPD. ${ }^{3} 1112$

The available data suggest considerable heterogeneity in the lower limb muscle fibre size and

\section{Key messages}

What is the key question?

- Are circulating muscle-specific microRNA levels different in patients with chronic obstructive pulmonary disease (COPD) compared with controls and are they associated with muscle biopsy characteristics?

\section{What is the bottom line?}

- MiR-1, miR-499, miR-133 and miR-206 levels are higher in patients with stable COPD compared with controls, likely as a result of the increase in muscle wasting and turnover which is associated with the development of skeletal muscle dysfunction in COPD.

\section{Why read on?}

- This study implies that altered muscle turnover is a component of stable COPD and that levels of circulating microRNAs reflect changes occurring within the skeletal muscle and may form part of a future diagnostic tool for COPD-associated muscle dysfunction.

proportions in patients with COPD. ${ }^{3} 13$ Potential therapeutic agents might address different facets of skeletal muscle dysfunction in COPD; in particular a drug which improved muscle bulk might not reverse fibre shift and vice versa, making biomarkers of skeletal muscle composition useful. ${ }^{14}$

The control of muscle mass and composition is complex, involving numerous transcription factors and other regulators including microRNAs. In muscle, much attention has focussed on miR-1, miR-499, miR-206, miR-133 (myomiRs) and miR-181. Some myomiRs affect myocyte proliferation and differentiation, and are organised as bicistronic genes, for example the miR-1 family. ${ }^{15}$ Others, for example miR-208b and miR-499, ${ }^{1516}$ are encoded within the slow myosin heavy chain (MHC) genes ${ }^{17}$ and thereby are restricted to type I fibres where they modulate fibre type. ${ }^{17}{ }^{18}$ Mir-181 is thought to maintain muscle mass in an activitydependent manner. We have previously shown that expression of miR-1 and miR-499 is suppressed in the quadriceps of patients with COPD. ${ }^{19}$

MiRNAs circulate in blood and are resistant to endogenous RNAses, ${ }^{20}$ therefore circulating 
miRNAs are potential disease biomarkers. ${ }^{21}$ We predicted that plasma levels of myomiRs differ between patients with COPD and age-matched healthy controls and are associated with fibre cross-sectional area (CSA) and fibre type proportions in patients. Furthermore, we investigated associations of plasma myomiRs with inflammatory signalling to provide insight into potential mechanisms of muscle wasting in COPD.

\section{METHODS}

Subjects

Patients with COPD according to the Global Initiative for Obstructive Lung Disease guidelines $2004^{22}$ (36 women, 67 men) were enrolled from clinics at the Royal Brompton Hospital (GOLD I or II, $\mathrm{n}=34$; III, $\mathrm{n}=38$; IV, $\mathrm{n}=31$ ). Patient exclusion criteria and ethical approval are described in the online supplement. Control subjects $(n=25)$ were recruited by local advertisement. Data on lung function, strength, fibre size, fibre proportion and MHC expression from this cohort have been published previously. ${ }^{19} 2324$

\section{Physiological measurements}

Measurements of lung volume, carbon monoxide transfer, blood gases, fat-free mass index (FFMI), quadriceps strength (maximum voluntary contraction (MVC)), and exercise performance (6 min walk distance (6MWD)) are described in the online supplement.

\section{Quadriceps muscle biopsy assessment}

Percutaneous needle biopsy of the vastus lateralis in the anterior mid-thigh of the leg on which strength was tested was performed under local anaesthesia using the Bergstrom technique. ${ }^{25}$ Fibre size and fibre proportion were determined by immunofluorescence. Messenger RNA (mRNA) was extracted and quantified as described previously ${ }^{26}$ and in the online supplement. Quadriceps nuclear factor $\kappa \mathrm{B}(\mathrm{NF} \kappa \mathrm{B}) \mathrm{p} 50$ and p65 subunits were analysed using transcription factor ELISA, also detailed in the online supplement.

\section{Measurement of circulating miRNA and cytokine levels}

Stored plasma samples were denatured, spiked with a synthetic RNA for normalisation, ${ }^{27}$ poly-adenylated and reverse transcribed and each miRNA was quantified by quantitative realtime PCR. Detailed methods are given in the online supplement. Cytokine levels were determined by luminex as described in the online supplement.

\section{Data analysis}

Data were analysed by Student's t test, ANOVA, Man-Whitney $\mathrm{U}$ test, Pearson correlation and receiver operating characteristic (ROC) analysis as appropriate and as described in the online supplement.

\section{RESULTS}

\section{Patient characteristics and muscle phenotype}

Consistent with a diagnosis of COPD, the patients had impaired lung function (forced expiratory volume in $1 \mathrm{~s}\left(\mathrm{FEV}_{1}\right)$ and gas transfer) and reduced arterial blood oxygen tensions compared with controls. There was no difference in age, body mass index, FFMI or arterial blood $\mathrm{CO}_{2}$ tensions between groups. Patients had a significantly reduced quadriceps force (MVC and twitch force) and exercise capacity measured by 6MWD compared with controls (table 1). Patients with GOLD III and GOLD IV

Table 1 Clinical characteristics of study subjects

\begin{tabular}{|c|c|c|c|}
\hline Condition & Control & COPD & Significance of difference, $p$ value \\
\hline Number & 25 & 103 & \\
\hline$M(F)$ & $14(11)$ & $67(36)$ & $0.4888(F)$ \\
\hline Age & $67.04(8.1)$ & $66.47(8.4)$ & $0.757(\mathrm{~T})$ \\
\hline Height $(\mathrm{cm})$ & $168(8.9)$ & $168(8.6)$ & $0.886(\mathrm{~T})$ \\
\hline Weight $(\mathrm{kg})^{*}$ & $69.1(83.5,62)$ & $68.8(80.6,59.5)$ & $0.359(\mathrm{MW})$ \\
\hline BMI $\left(\mathrm{kg} / \mathrm{m}^{2}\right)^{*}$ & $24.8(27.05,24)$ & $24.1(27,21.4)$ & $0.134(\mathrm{MW})$ \\
\hline FFMI $\left(\mathrm{kg} / \mathrm{m}^{2}\right)^{*}$ & $16.4(19.8,15.2)$ & $15.7(17.2,14.5)$ & $0.060(\mathrm{MW})$ \\
\hline Pack year smoking* & $0(8.75,0)$ & $45(60.6,34)$ & $<0.001(\mathrm{MW})$ \\
\hline $\mathrm{FEV}_{1}(\% \text { pred })^{*}$ & $110(114,101)$ & $42(57,27)$ & $<0.001(\mathrm{MW})$ \\
\hline RV/TLC (\%) & $37(4.8)$ & $57(9.2)$ & $<0.001(\mathrm{~T})$ \\
\hline $\mathrm{TL}_{\mathrm{CO}}(\%$ pred $)$ & $91(14.2)$ & 43 (17.6) & $<0.001(\mathrm{~T})$ \\
\hline $\mathrm{PaCO}_{2}(\mathrm{kPa})$ & $5.28(0.4)$ & $5.14(0.6)$ & $0.337(\mathrm{~T})$ \\
\hline $\mathrm{PaO}_{2}(\mathrm{kPa})$ & $11.0(1.2)$ & $9.31(1.3)$ & $<0.001(\mathrm{~T})$ \\
\hline 6MWD (m) & $614(83)$ & 394 (120). & $<0.001(\mathrm{~T})$ \\
\hline SGRQ* & $2(7.5,0)$ & $54(64,43)$ & $<0.001(\mathrm{MW})$ \\
\hline Best MVC $(\mathrm{kg})$ & $35.8(10.2)$ & $29.5(10.2)$ & $0.006(\mathrm{~T})$ \\
\hline Best TwQ $(\mathrm{kg})^{*}$ & $8.6(11.4,7.0)$ & $7.6(9.2,6)$ & $0.081(\mathrm{MW})$ \\
\hline MVC/wt $(\mathrm{kg} / \mathrm{kg})^{*}$ & $0.49(0.38,0.57)$ & $0.41(0.37,0.51)$ & $0.014(\mathrm{MW})$ \\
\hline $\mathrm{MHCl}$ mRNA $(\mathrm{AU})^{*}$ & $32(48,20)$ & $7.3(14.5,4.6)$ & $<0.001(\mathrm{MW})$ \\
\hline MHClla mRNA $(A U)^{*}$ & $1.6(2.3,0.9)$ & $2.5(3.8,1.5)$ & $0.045(\mathrm{MW})$ \\
\hline MHCIIx mRNA (AU)* & $5.2(8.1,4.2)$ & $11.4(19.3,8.0)$ & $<0.001(\mathrm{MW})$ \\
\hline
\end{tabular}

Values are mean (SD) for normally distributed data or median (75th, 25th percentile) for non-normally distributed data in patients with COPD or healthy controls (indicated by ${ }^{*}$. $\mathrm{p}$ Values were calculated by t test (normally distributed data, T), Mann-Whitney test (non-normally distributed data, MW) or Fischer's exact test (F) and are shown in bold when $\mathrm{p}<0.05$. MHC RNAs were determined by quantitative PCR and normalised to the expression of RPLPO in the same samples as described in the online supplement.

6MWD, 6 min walk distance; BMI, body mass index; COPD, chronic obstructive pulmonary disease; CSA, cross-sectional area; FEV ${ }_{1}$, forced expiratory volume in 1 s; FFMI, fat-free mass index; $\mathrm{MHC}$, myosin heavy chain; $\mathrm{MVC}$, maximal voluntary contraction; $\mathrm{PaCO}_{2}$, arterial carbon dioxide tension; $\mathrm{PaO}_{2}$, arterial oxygen tension; pred, predicted; $\mathrm{RV}$, residual volume;

SGRQ, St George's Respiratory Questionnaire; TLC, total lung capacity; $\mathrm{TL}_{\mathrm{CO}}$, transfer factor of the lung for carbon monoxide; TwQ, twitch force in the quadriceps. 


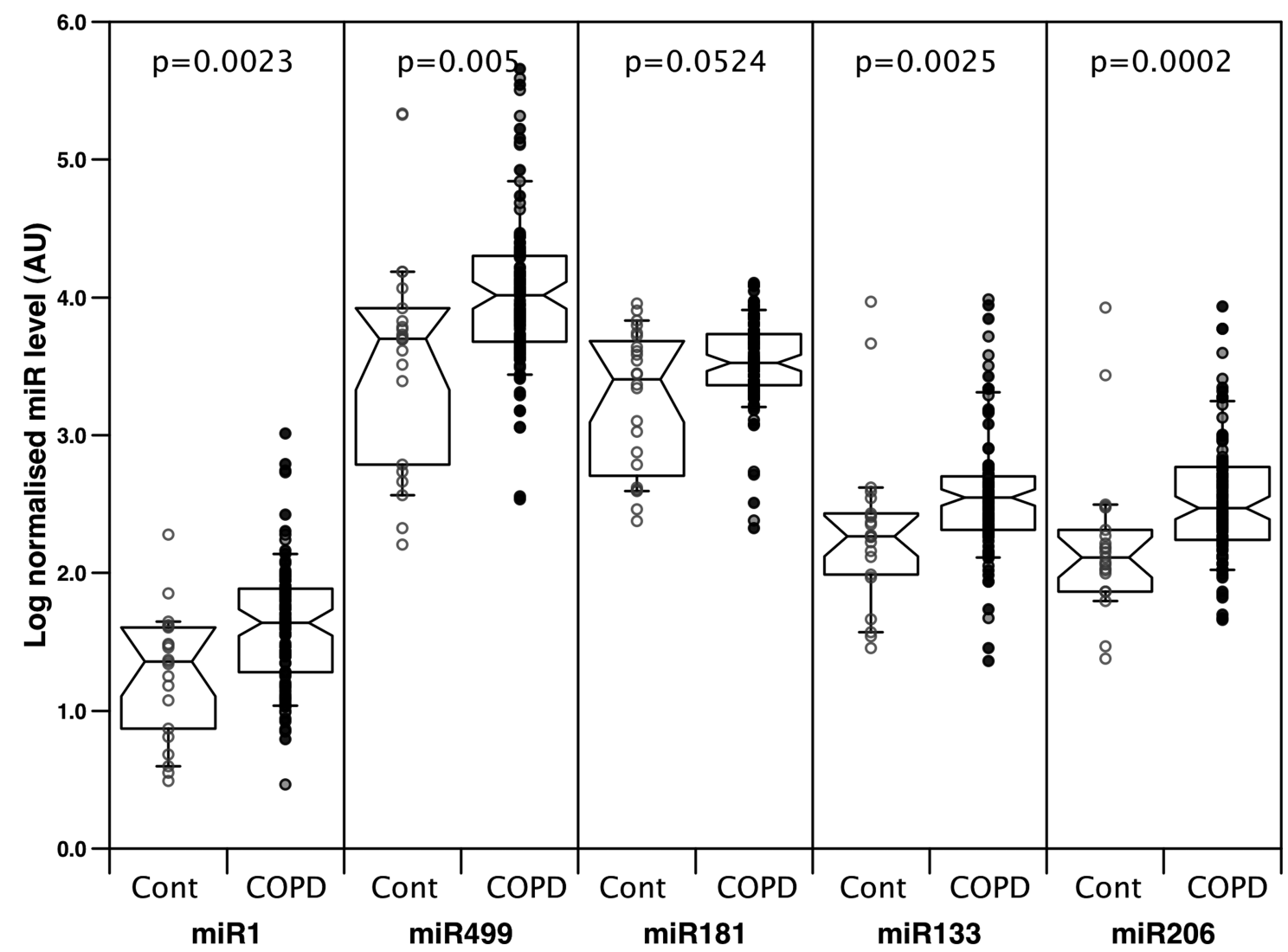

Figure 1 Muscle-derived miRNAs are elevated in the plasma of patients with stable chronic obstructive pulmonary disease (COPD). Plasma miR levels were measured as described in 'Methods' and normalised to a spiked Caenorhabditis elegans control. Data are presented as log-normalised levels with the notched boxes showing median and IQR, error bars at the 10th and 90th percentile, outliers are also shown. Patients with Global Initiative for Obstructive Lung Disease (GOLD) stage I and II COPD are represented by filled grey circles, those with GOLD stage III and IV disease are represented by filled black circles and controls are shown as open grey circles. Statistical significance was calculated by t test or Mann-Whitney $U$ test as the non-parametric alternative.

COPD had significantly lower FFMI than those with GOLD II COPD and patients with GOLD IV COPD had significantly worse 6MWD compared with those with GOLD II or III. Quadriceps strength did not differ significantly across the GOLD stages (see online supplementary table S1, figure S1).

Quadriceps muscle fibre size and proportion for the entire cohort have been reported. ${ }^{3}$ The association of fibre type and size with GOLD stage for those included in this study is shown in online supplementary table S1. From that report we highlight that the patients had markedly smaller IIX fibres $(p<0.001)$, more type II fibres and fewer type I fibres. Neither fibre proportion nor fibre CSA was different between the GOLD groups (see online supplementary table S1).

\section{MiRNA levels in the plasma of patients with COPD compared with control subjects}

Circulating levels of miR-1, miR-499, miR-206 and miR-133 were significantly elevated in the plasma of patients with COPD compared with those in controls (figure 1). MiR-1 and miR-499 had the greatest fold difference, 2.5 and 1.5 times higher in patients with COPD and controls respectively. The trend towards an increase in miR-181 did not reach significance $(p=0.052)$. By contrast there was no difference in levels of miR-16 (an miRNA previously used as a control in plasma ${ }^{27}$ ) or miR-122 (a liver-specific miRNA $^{28}$ ), both serving as non-muscle-specific miR controls (see online supplementary figure S2) or in the spiked in exogenous control raw cycle threshold values in patients compared with controls $(p=0.308)$. As smoking affects miRNA levels in the lung we compared plasma levels of the miRNAs in current $(n=16)$ and ex-smokers $(n=87)$ with COPD; smoking status did not affect plasma miRNA levels significantly.

\section{Plasma microRNA and lung function}

MiR-1 was negatively but modestly associated with $\mathrm{FEV}_{1}$ as $\%$ predicted $(\mathrm{r}=-0.3, \mathrm{p}=0.001)$ and with the transfer factor of the lung for carbon monoxide $\left(\mathrm{TL}_{\mathrm{co}}\right) \%$ predicted $(\mathrm{r}=-0.3$, $\mathrm{p}=0.002$ ) when all subjects were analysed (figure 2). MiR-499, miR-206 and miR-133 showed a distinct and similar pattern. Although plasma miRNA was higher in patients than in the controls, within the patient group, the miRNAs were higher in patients with the most preserved lung function (figure 2; see also online supplementary figure S3). Patients with GOLD IV COPD had lower plasma miR-499 and miR-206 than those with GOLD II COPD $(p=0.004$ and $p=0.007$ respectively; see online supplementary figure S3) and both miRNAs had a modest positive correlation with $\mathrm{FEV}_{1} \%$ predicted within the patients alone (miR-499 $\mathrm{r}=0.26, \mathrm{p}=0.007$; miR-206 $\mathrm{r}=0.25$, $\mathrm{p}=0.013$; figure 2). Neither miR-16 nor miR-122 varied with GOLD stage (see online supplementary figure S4). Although there were no overt differences in miRNA levels between smokers and ex-smokers with COPD, because of the potential confounder of smoking status, we re-analysed these correlations in ex-smoking patients alone and found that the correlation was 
A
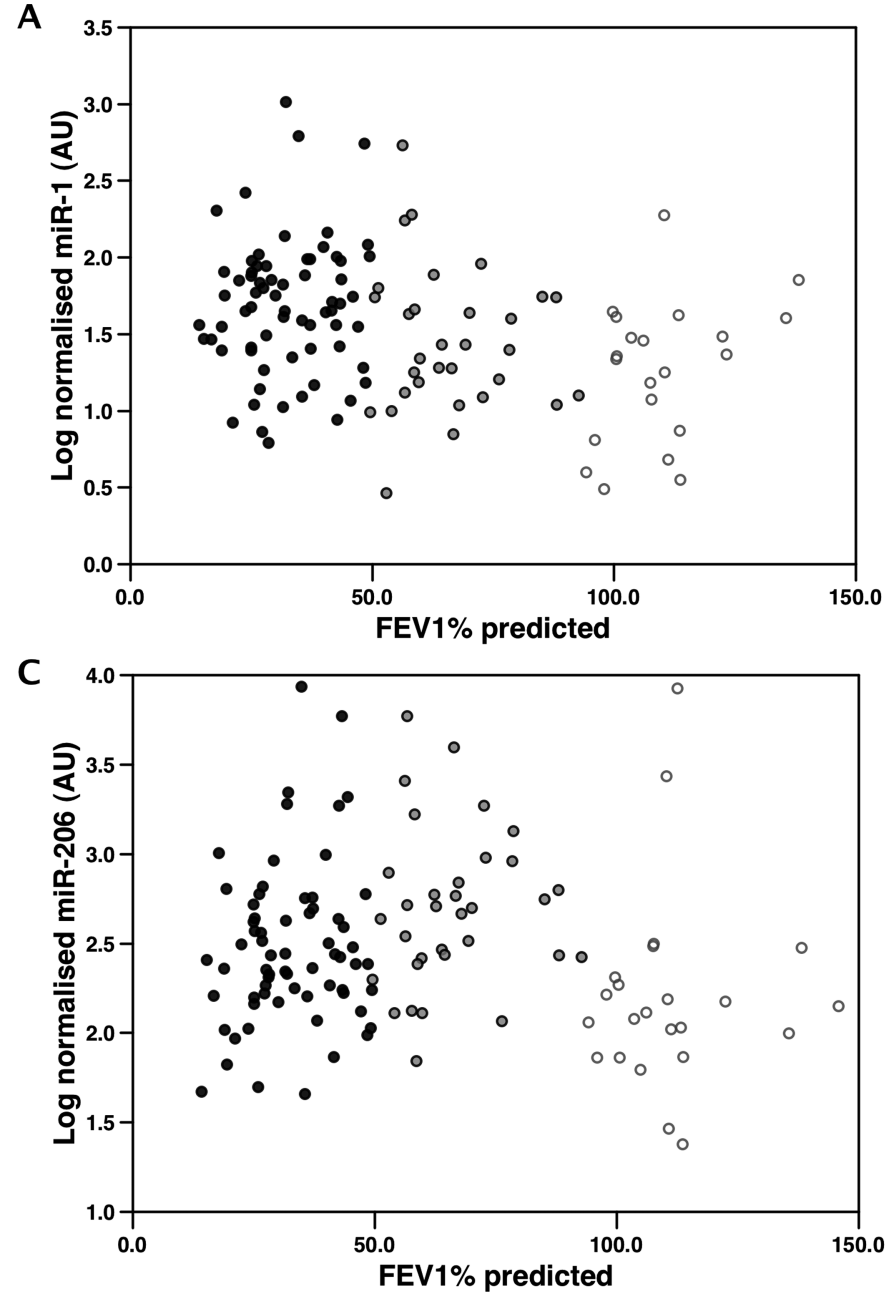

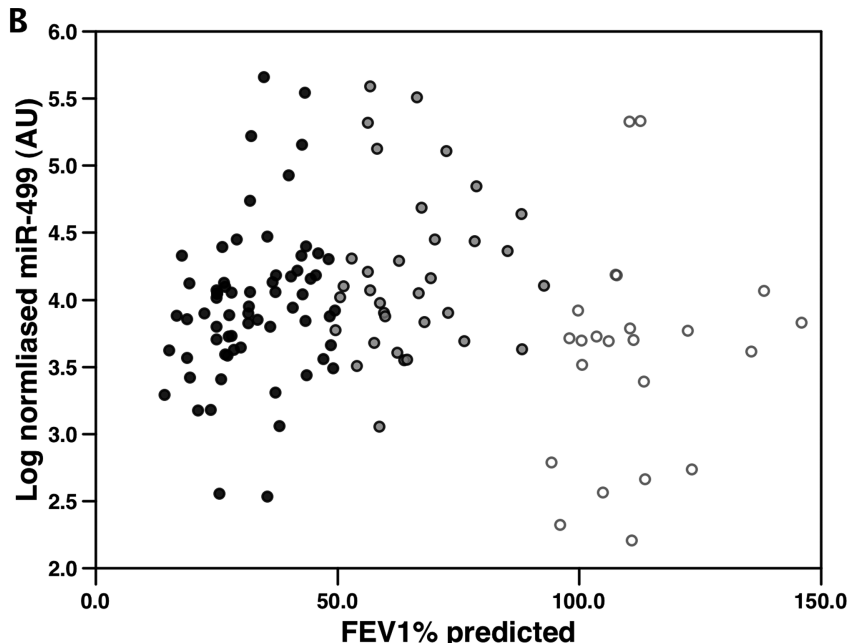

FEV1\% predicted

Figure 2 Association of plasma miRNAs with lung function. Plasma miRNAs were determined as described in 'Methods' and compared with forced expiratory volume in $1 \mathrm{~s}\left(\mathrm{FEV}_{1}\right) \%$ predicted. MiR-1 showed a negative association with $\mathrm{FEV}_{1}$ across the whole cohort $(A)$ whereas miR-499 (B) and miR-206 (C) were highest in patients with early stage chronic obstructive pulmonary disease (COPD) (Global Initiative for Obstructive Lung Disease (GOLD) I and II). Consequently although miR-499 and miR-206 levels were significantly higher in patients than controls (see figure 1) there was a negative association of these miRNAs with $\mathrm{FEV}_{1}$ within the patients group alone (miR-499 $r=0.29, p<0.003 ; \mathrm{miR}-206 \mathrm{r}=0.26, \mathrm{p}<0.009$ ). Patients with GOLD I and II COPD are represented by filled grey circles, those with GOLD III and IV COPD are represented by filled black circles and control subjects are represented by open grey circles.

numerically strengthened despite a smaller number of data points (miR-499 r=0.3, $\mathrm{p}=0.006$; miR-206, $\mathrm{r}=0.34 \mathrm{p}=0.001$ ).

\section{Plasma microRNA, muscle mass and strength}

Plasma miR-1 levels negatively correlated with FFMI when patients were analysed either with or without the controls $(r=-0.21$, $\mathrm{p}=0.036$ and $\mathrm{r}=-0.25, \mathrm{p}=0.013$, respectively; figure 3 ) and also correlated with type I fibre CSA in patients $(r=-0.27, p=0.027$; figure 3). In ex-smoking patients FFMI was more strongly associated with plasma miR-1 than in the entire patient cohort $(\mathrm{r}=-0.30, \mathrm{p}=0.005$; see online supplementary figure $\mathrm{S} 5)$. However, there was no association of miR-1 with strength.

The other myomiRs were not associated with FFMI or with fibre CSA and showed only weak correlations between circulating miR levels and measures of quadriceps strength. MiR-133, miR-499 and miR-206 weakly correlated with quadriceps MVC corrected for weight $(r=0.23, p=0.022 ; r=0.20, p=0.036$; and $\mathrm{r}=0.21, \mathrm{p}=0.034$, respectively). In ex-smoking patients the associations were stronger than in the whole cohort $(r=0.29$, $\mathrm{p}=0.008 ; \mathrm{r}=0.27, \mathrm{p}=0.013$; and $\mathrm{r}=0.28, \mathrm{p}=0.009$; see online supplementary figure S5).

\section{Plasma miRNAs and fibre shift}

Reduced lung function indices are associated with a reduction in the percentage of type I fibres (TI\%). In the entire patient group none of the miRNAs were associated with TI\%. However, restricting the patient group to those with GOLD III and IV disease showed a weak association between plasma miR-499 and TI\% $(r=0.26, p=0.033)$ and plasma miR-499 was significantly lower in those with evidence of pathological fibre shift (figure 4). MiR-499 was positively correlated with 6MWD $(\mathrm{r}=0.22, \mathrm{p}=0.028$; figure 4$)$, an association numerically strengthened by considering ex-smoking patients alone $(r=0.25$, $\mathrm{p}=0.020$ ). However, miR-499 was not correlated with quadriceps endurance.

To determine whether plasma myomiRs are useful predictors of fibre-type shift, we performed ROC analysis which showed that $\mathrm{TL}_{\mathrm{CO}} \%$ predicted and $6 \mathrm{MWD} \%$ predicted were better predictors of fibre shift than the plasma miRNAs alone in patients with GOLD III and IV COPD, but combining these values, the ROC area under the curve (AUC) increased to 0.89 (table 2, figure 5 and see online supplementary figure S6). Inclusion of patients with GOLD I and II COPD (who did not show an association between 
A

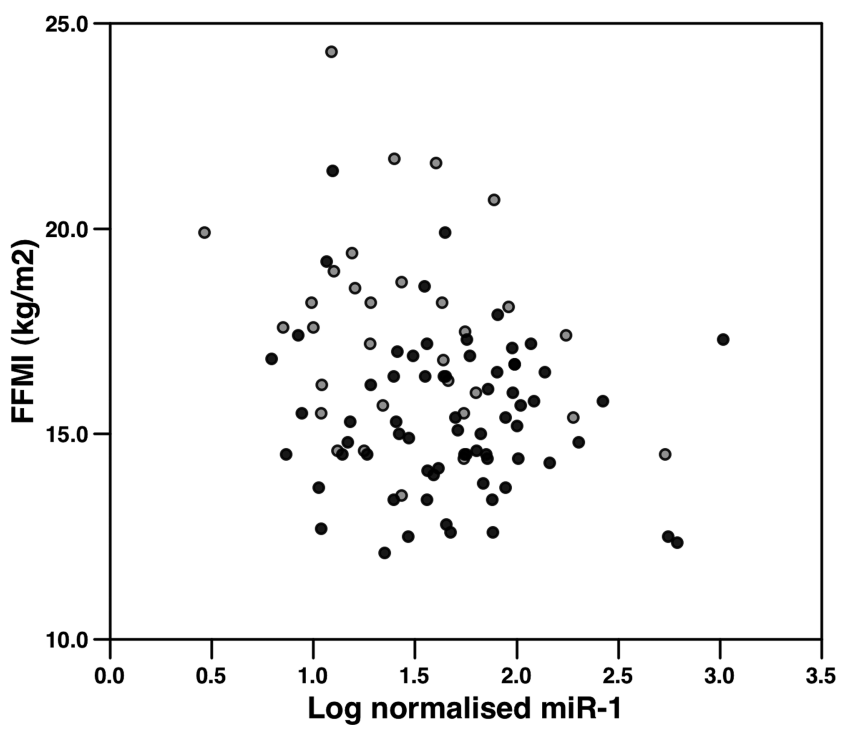

B

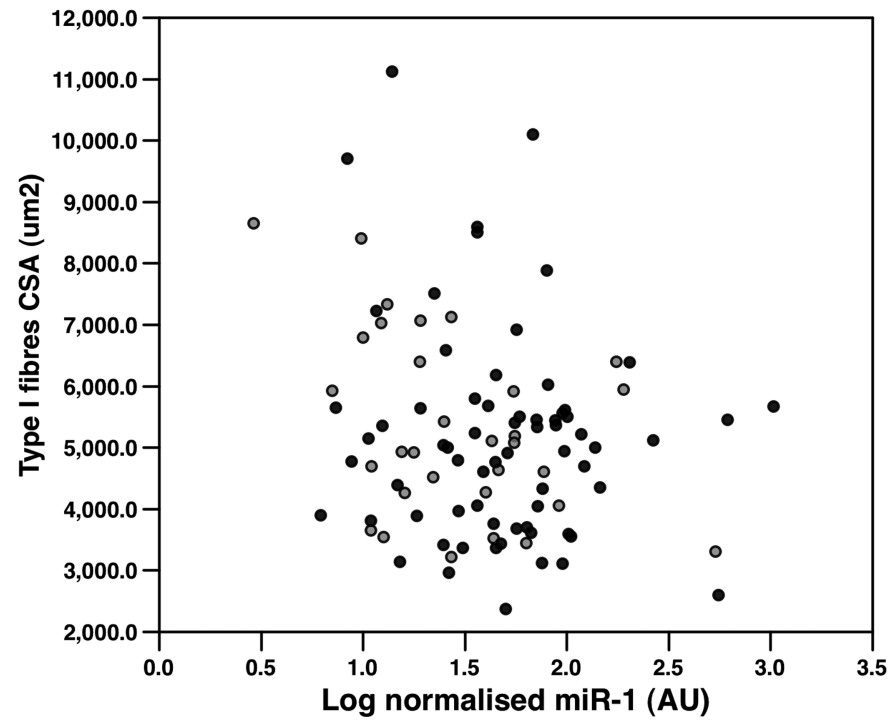

Figure 3 Plasma miR-1 is weakly associated with fat-free mass and type I fibre cross-sectional area (CSA) in patients with chronic obstructive pulmonary disease (COPD). Physiological parameters and plasma miRNA levels were determined as described in 'Methods'. (A) Plasma miR-1 levels negatively correlated with fat-free mass index when patients with COPD were analysed either with or without the controls (as shown in the figure), $r=-0.21, p=0.036$ and $r=-0.25, p=0.013$, respectively. (B) Plasma miR-1 levels were negatively correlated with type I fibre CSA in patients $(r=-0.27, p=0.027)$. Patients with Global Initiative for Obstructive Lung Disease (GOLD) I and II COPD are represented by filled grey circles, those with GOLD III and IV COPD are represented by filled black circles.

$\mathrm{TI} \%$ and plasma miR-499) reduced the predictive value of physiological and miRNA values for fibre shift (eg, $\mathrm{TL}_{\mathrm{CO}} \%$ predicted $\mathrm{AUC}=0.70$ and the combined score $\mathrm{AUC}=0.79$ ).

\section{Plasma miR-499 with markers of inflammation}

To investigate potential mechanisms contributing to the increase in plasma myomiRs we compared $\mathrm{NF \kappa B}$ p 50 and $\mathrm{NF \kappa B}$ p 60 in nuclei prepared from quadriceps muscle with plasma myomiR levels. Overall, there was no difference in NFKB p65 and p50 binding DNA in patients compared with controls $(\mathrm{p}=0.134$ and $\mathrm{p}=0.968$, respectively; see online supplementary table S1), and there was no relationship of these factors with muscle mass, fibre type or function. However, miR-499 levels were found to correlate weakly with nuclear $\mathrm{NF} \kappa \mathrm{B}$ p50 $(\mathrm{r}=0.198, \mathrm{p}=0.028)$. This association persisted when only patients with COPD were considered $(\mathrm{r}=0.23, \mathrm{p}=0.021)$. Restricting the patients to GOLD I and II only strengthened the association of nuclear $\mathrm{NF \kappa B}$ p50 with miR-499 ( $r=0.58, p<0.001)$, while an association was absent in patients with GOLD III and IV COPD $(r=0.142$, $\mathrm{p}=0.255$; figure 6). While miR-1 did not correlate with nuclear NF-kB p50 in any group, miR-133 $(r=0.47, p=0.006)$ and miR-206 $(r=0.4, p=0.016)$ were associated with nuclear $\mathrm{NF \kappa B}$ p50 in patients with GOLD I and II COPD. There were no associations of plasma miRNAs with nuclear NFкB p65.

Plasma myomiR levels were also compared with circulating levels of tumour necrosis factor $\alpha$, interleukin 2 (IL2) and IL5. In the patients alone, plasma levels of miR-206, miR-133 and miR-499, but not miR-1, were associated with plasma levels of the cytokines (figure 7, table 3), in particular in patients with GOLD III and IV disease. The strongest associations between the circulating cytokines and myomiRs were with miR-206 and the weakest associations were with miR-499 (figure 7).

\section{DISCUSSION}

The main finding of this study is that, in patients with stable COPD, plasma levels of muscle-specific miRNAs but not of two control miRNAs are increased, suggesting that muscle wasting or turnover is increased even in those with stable COPD. Furthermore, we show that different miRNAs have distinct plasma profiles dependent on disease severity and on the proportion of oxidative fibres remaining in the quadriceps. Alone these miRNAs show weak discrimination of patients with type I fibre shift, but they improve the utility of $\mathrm{TL}_{\mathrm{CO}} \%$ predicted and 6MWD\% predicted to identify patients with pathological type I fibre shift in those with GOLD III and IV disease. Finally we show that in mild/moderate disease, plasma levels of miR-499 are associated with nuclear $\mathrm{NF \kappa B}$ p50 but not $\mathrm{NF \kappa B}$ p65, whereas in severe/very severe disease, miR-206 and miR-133 are associated with circulating cytokines. These data raise the possibility that wasting in mild disease is predominantly driven by inactivity but in severe disease is associated with systemic inflammation.

\section{Significance of the findings}

The purpose of this study was to identify biomarkers and mechanisms of skeletal muscle dysfunction and fibre abnormalities in COPD.

\section{Disease process and mechanism}

Increased plasma myomiRs are associated with wasting in animals and humans. For example, in patients with Duchenne muscular dystrophy and in $m d x$ mice serum levels of miR-1, miR-133 and miR-206 are elevated, ${ }^{29}$ suggesting that the increase in plasma myomiRs observed in our cohort derives from atrophying muscle or muscle with elevated turnover. As our patients were in the stable phase of COPD, these data indicate that increased muscle turnover occurs in patients with COPD in the absence of current or recent exacerbation.

Although plasma levels of miR-499, miR-133 and miR-206 were elevated in patients compared with controls, within the patient group the highest plasma levels were associated with better lung function. This apparent paradox could be explained 
A

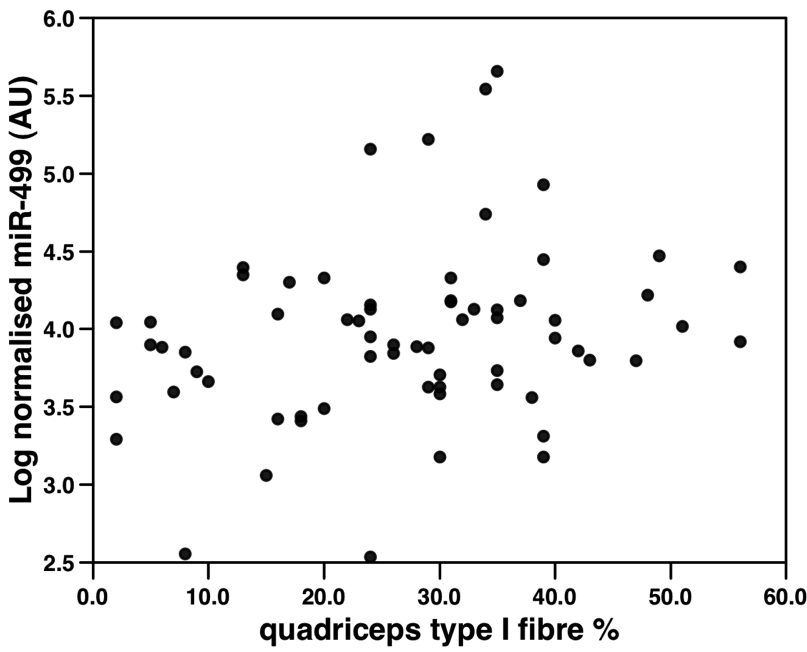

C

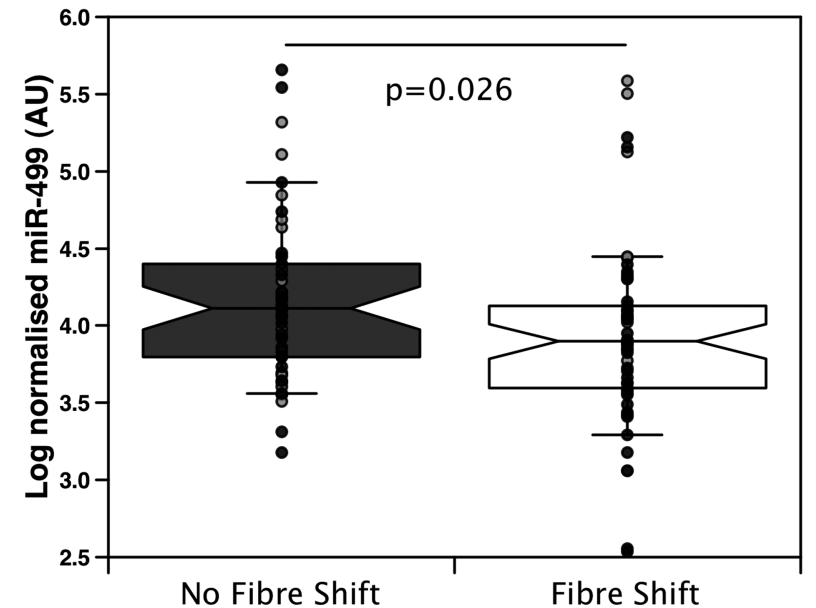

B

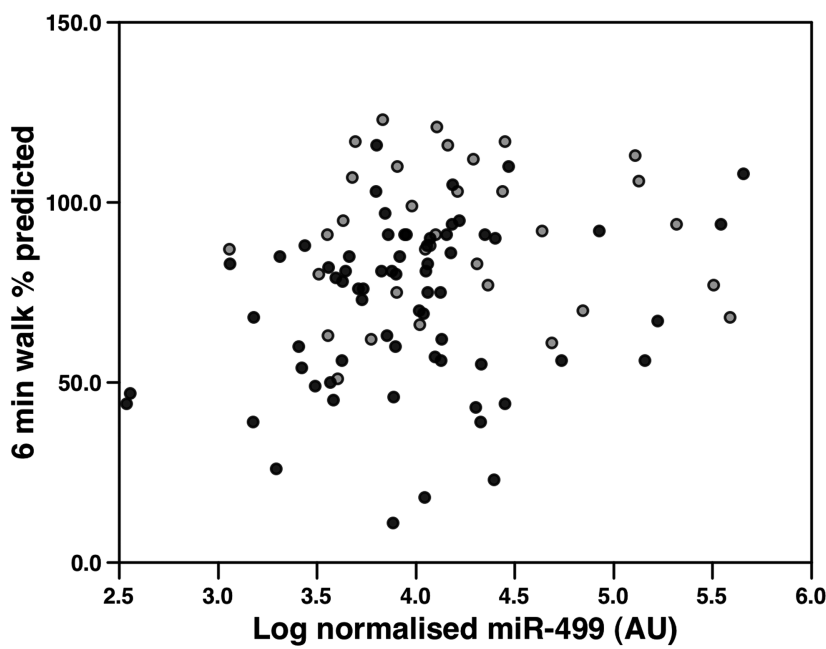

Figure 4 Association of plasma miR-499 and fibre proportion in patients with Global Initiative for Obstructive Lung Disease (GOLD) III and IV chronic obstructive pulmonary disease (COPD). Plasma miR-499 was determined as described in 'Methods'. In the entire patient group none of the miRNAs were associated with the percentage of type I fibre (TI\%) in quadriceps muscle biopsy. (A) Comparison of fibre proportion and plasma miR-499 levels in patients with GOLD III and IV COPD showed a weak association of plasma miR-499 with TI\% in quadriceps biopsies ( $r=0.26$, $p=0.033$ ). (B) In the entire patient cohort, comparison of plasma miR-499 in patients with fibre shift (defined as a Tl\% below the 2.5 th percentile for the control cohort) with those without fibre shift had higher plasma miR-499. Median (25th, 75th percentile) log $A U=4.113(3.781,4.412)$ and $3.898(3.594,4.126)$ respectively (Mann-Whitney test). Error bars are 10th percentile and 90th percentile and outliers are shown. (C) Comparison of 6 min walk distance with plasma miR-499 showed a weak association in the entire patient cohort $(r=0.22, p=0.028)$. Patients with GOLD I and II COPD are represented by filled grey circles and those with GOLD III and IV COPD are represented by black circles.

Table 2 ROC analysis multiplied by -1 , predicting presence of type I fibre shift

\begin{tabular}{|c|c|c|c|c|c|c|c|}
\hline $\mathrm{TL}_{\mathrm{CO}} \%$ & $6 \mathrm{MWD} \%$ pred & miR-1 & miR-499 & miR-133 & miR-206 & miR-181 & AUC \\
\hline \multirow[t]{6}{*}{+} & & & & & & & 0.79 \\
\hline & + & & & & & & 0.78 \\
\hline & & + & & & & & 0.63 \\
\hline & & & + & & & & 0.71 \\
\hline & & & & + & & & 0.69 \\
\hline & & & & & + & + & 0.66 \\
\hline & & & & & & & 0.61 \\
\hline+ & + & & & & & & 0.83 \\
\hline+ & & + & & & & & 0.84 \\
\hline+ & & & + & & & & 0.83 \\
\hline+ & & + & + & & & & 0.86 \\
\hline+ & + & + & + & & & & 0.87 \\
\hline+ & + & + & + & & + & + & 0.89 \\
\hline
\end{tabular}

Components included in each ROC analysis are indicated by + .

6MWD, 6 min walk distance; AUC, area under the curve; pred, predicted; ROC, receiver operating characteristic; $\mathrm{TL}_{\mathrm{CO}}$, transfer factor of the lung for carbon monoxide. 

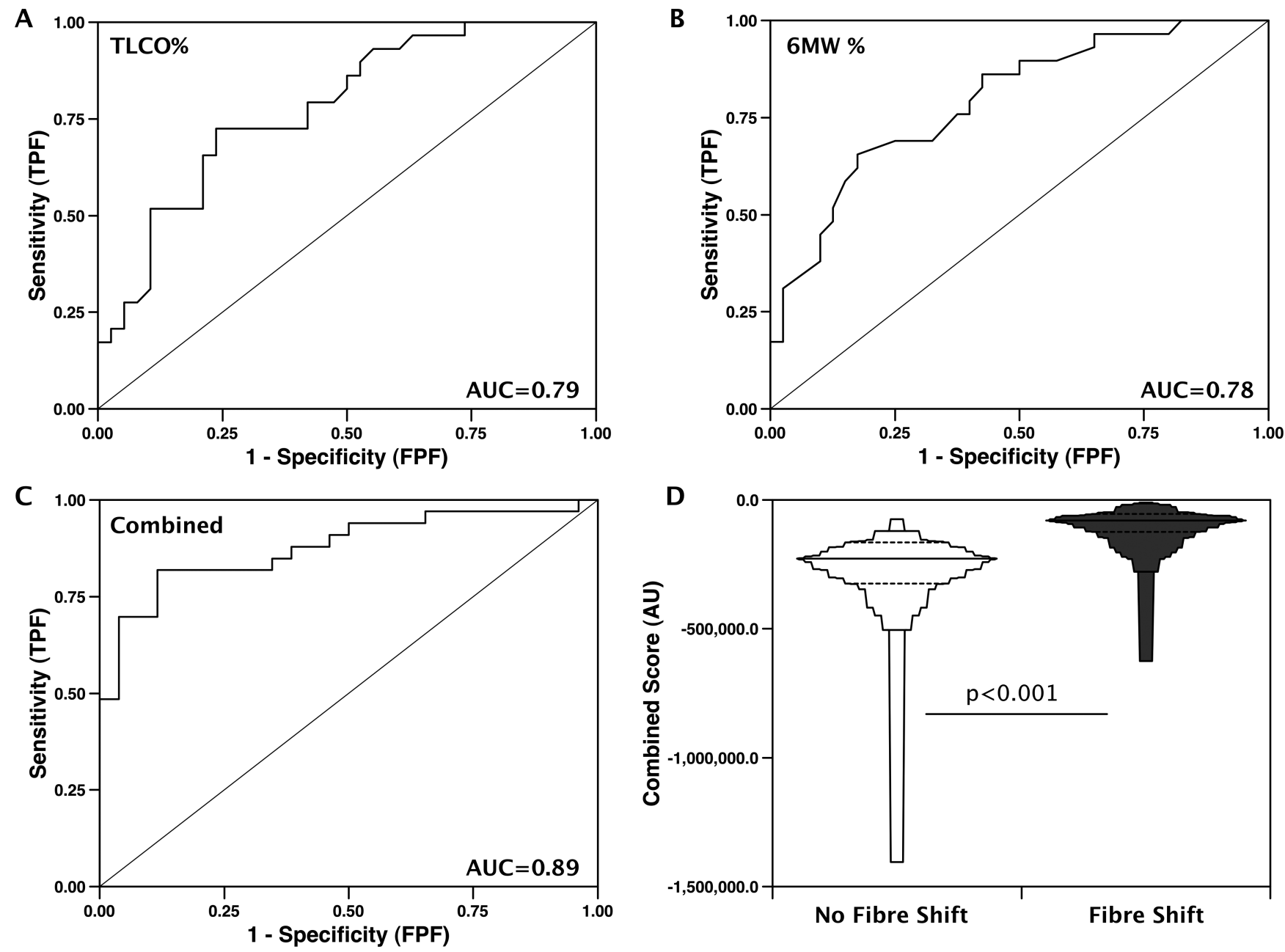

Figure 5 Receiver operating characteristic (ROC) analyses of physiological parameters alone or in combination with plasma miRNA levels to discriminate patients according to the presence of type I fibre shift. Patients with Global Initiative for Obstructive Lung Disease (GOLD) III and IV disease were defined as having type I fibre shift or not as described in 'Results'. ROC analysis was then performed as described in 'Methods' using transfer factor of the lung for carbon monoxide ( $\left.\mathrm{TL}_{\mathrm{CO}}\right)$ expressed as \% predicted $(\mathrm{A}), 6$ min walk distance $(6 \mathrm{MWD})$ as \% predicted (B), combination score (generated by combining $\mathrm{TL}_{\mathrm{CO}} \%$ predicted, 6MWD \% predicted, plasma miR-1 level, plasma miR-499 level, plasma miR-181 level and plasma miR-206 level, as described in 'Methods') (C). Area under the curve (AUC) is shown in each graph. (D) Comparison of the combined score in patients with or without fibre shift. Patients with GOLD III and IV disease with fibre shift have a significantly different score $(p<0.001)$ than patients of the same disease severity without fibre shift. TPF, true positive fraction.

by differences in the muscle pool in patients with mild versus severe disease. Patients with severe disease have a lower muscle mass from which to release miRNA compared with patients with mild disease. Also, patients with more severe disease have a lower proportion of type I fibres from which to release miR-499 compared with patients with mild disease, miR-499 being encoded within a slow MHC gene and so only expressed in type I fibres. ${ }^{17}$ The decrease in miR-499 observed in patients with severe lung function impairment is consistent with observations from patients with lung cancer in whom low plasma levels of miR-499 were a predictor of reduced survival, ${ }^{30}$ perhaps as a result of cachexia.

Our data also show that in mild COPD, plasma miR-499 levels are directly correlated with nuclear NFкB p50. This observation allows us to tentatively speculate that loss of type I fibres or export of miR-499 is dependent on NFKB activation. However, as plasma miR-499 levels were not associated with NFкB p65, the data suggest that the increase in plasma miR-499 is associated with the non-canonical $\mathrm{NF \kappa B}$ pathway that utilises $\mathrm{NF \kappa B}$ p50 and $\mathrm{BCl} 3$ in response to physical inactivity as demonstrated by transgenic mouse models. ${ }^{31}$ Alternatively, since miRs can be exported in an active and selective manner, ${ }^{32}$ it is possible that NFKB p50 drives the export of miR-499 from type I fibres. Given the role of miR-499 in type I fibre maintenance, directed secretion of miR-499 may be required for fibre switching in response to inactivity. Again this process would be most active in the earlier stages of the disease when there are more type I fibres. Plasma cytokines were also associated with plasma myomiRs, in particular miR-206 and miR-133 in patients with severe disease. This observation raises the possibility that inflammation is an important driver of wasting in this patient subgroup. Alternatively, as there was no association of the plasma myomiRs and nuclear NFKB p65, the plasma myomiRs and inflammatory cytokines could be associated in a NFKB-independent manner.

\section{Plasma myomiRs as diagnostic tools in COPD-associated fibre-type abnormalities}

Muscle biopsy is the only way to investigate fibre-type changes but is invasive, introducing discomfort and inconvenience to the patient. Therefore, it would be very useful to identify a less 

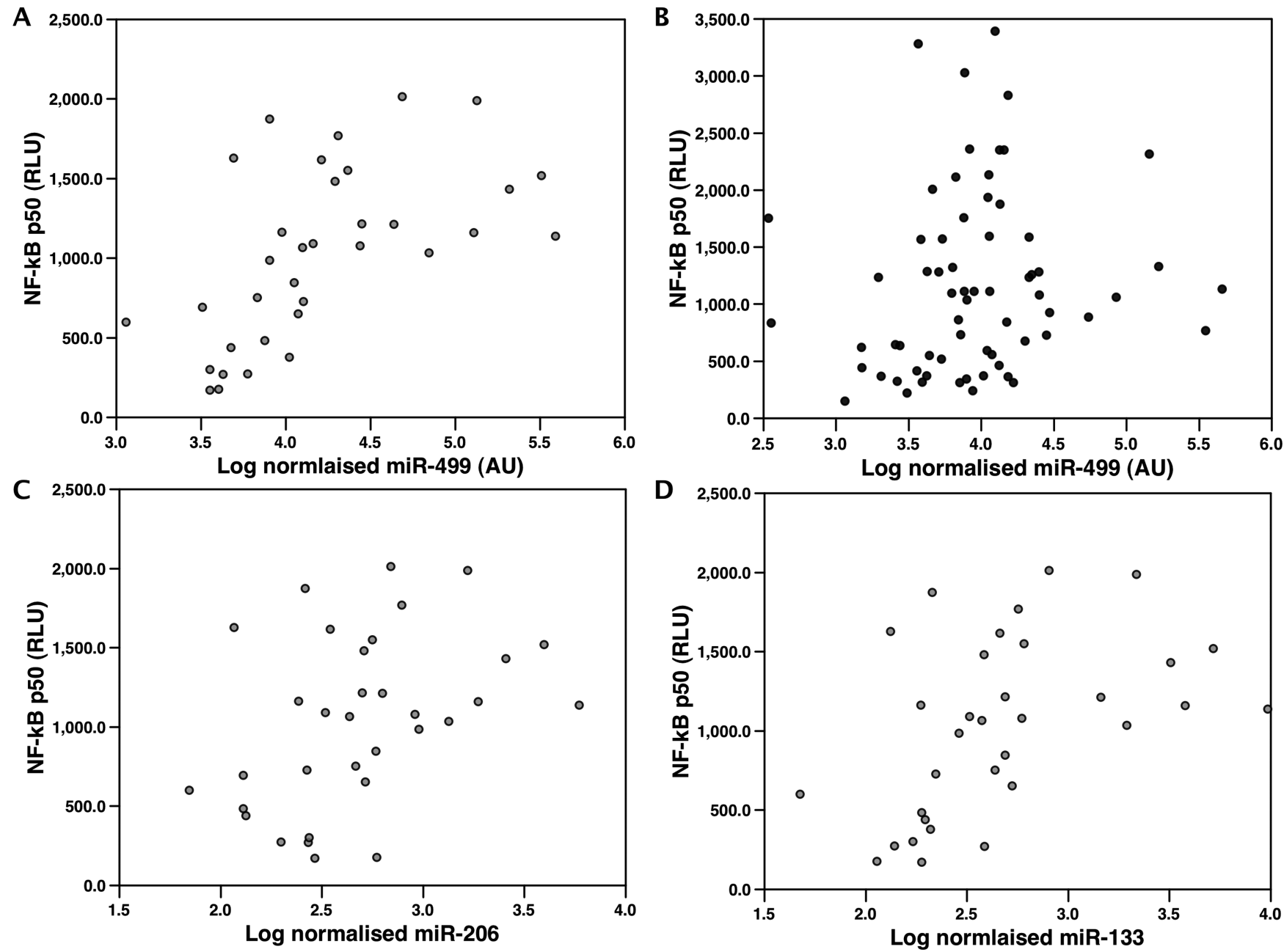

Figure 6 Plasma miR-499, miR-133 and miR-206 are correlated with the amount of nuclear factor $\kappa B(N F \kappa B)$ in the quadriceps nuclei of patients with Global Initiative for Obstructive Lung Disease (GOLD) I and II chronic obstructive pulmonary disease (COPD). Log normalised plasma miR-499 (determined as described in 'Methods') was compared with NFKB p50 determined by transcription factor ELISA. In patients with GOLD I and II COPD there was a direct association between NFKB p50 $(r=0.58, p<0.001)$ and miR-499 (A) that was not present when analysing patients with GOLD III and IV COPD ( $r=0.142, p=0.25$ (B). In patients with GOLD I and II COPD NFKB p50 was also correlated with miR-206 ( $r=0.4 p=0.016$ (C) and miR-133 ( $r=0.47, p=0.006$ (D). Patients with GOLD I and II COPD are represented by filled grey circles, those with GOLD III and IV COPD are represented by filled black circles.

invasive measure of muscle fibre-type abnormalities. Alone, neither the miRNAs studied here nor the physiological parameters are sufficient to identify patients with a significant fibre shift. However, by combining these factors we were able to improve discrimination for identifying type I fibre shift. This approach only worked in the severe disease groups (GOLD III and IV), presumably because the more dynamic changes in quadriceps mass have already occurred. ${ }^{7}$ Whilst not sufficient to replace muscle biopsy based on the present data, increasing the number of miRNAs profiled has potential to improve the predictive power further, while a larger study would increase the power to detect a potential biomarker from the targets already studied.

\section{Critique of the method}

The myomiRs we evaluated are known to be highly enriched in muscle, suggesting that the majority of myomiRs measured in plasma are of muscle origin. However, with the exception of miR-206 these miRNAs could come from either cardiac or skeletal muscle; miR-206 is almost exclusively expressed in skeletal muscle and is not readily detectable in the heart by RNAse protection $^{33}$ or real-time PCR. ${ }^{34}$ Given that miR-133 and miR-499 show very similar patterns in patients with COPD to that of miR-206, they are also likely to be of skeletal muscle origin. MiR-1 plasma levels had a linear correlation with $\mathrm{FEV}_{1}$, unlike the other myomiRs and miR-1 was present at the lowest levels. Cardiac muscle is another potential source of miR-1, miR-133 and miR-499, but studies have failed to demonstrate major changes in circulating myomiRs in chronic heart failure, diastolic dysfunction or hypertrophy, ${ }^{35}$ suggesting that the heart is an unlikely contributor to the elevation of plasma myomiRs in patients with COPD. It is not possible to determine the proportion of each myomiR that is derived from individual muscles. However, the quadriceps muscle contributes approximately $40 \%$ of the skeletal muscle bulk and is known to be the predominant muscle affected in patients with COPD, even within the lower limb. ${ }^{36}$ If the diaphragm was the major source of the elevated plasma myomiRs, we would have predicted an increase in miR-499 as a result of the increase in TI\% in the diaphragm in patients with COPD compared with controls, and potentially an increase in miR-499 with advancing GOLD 

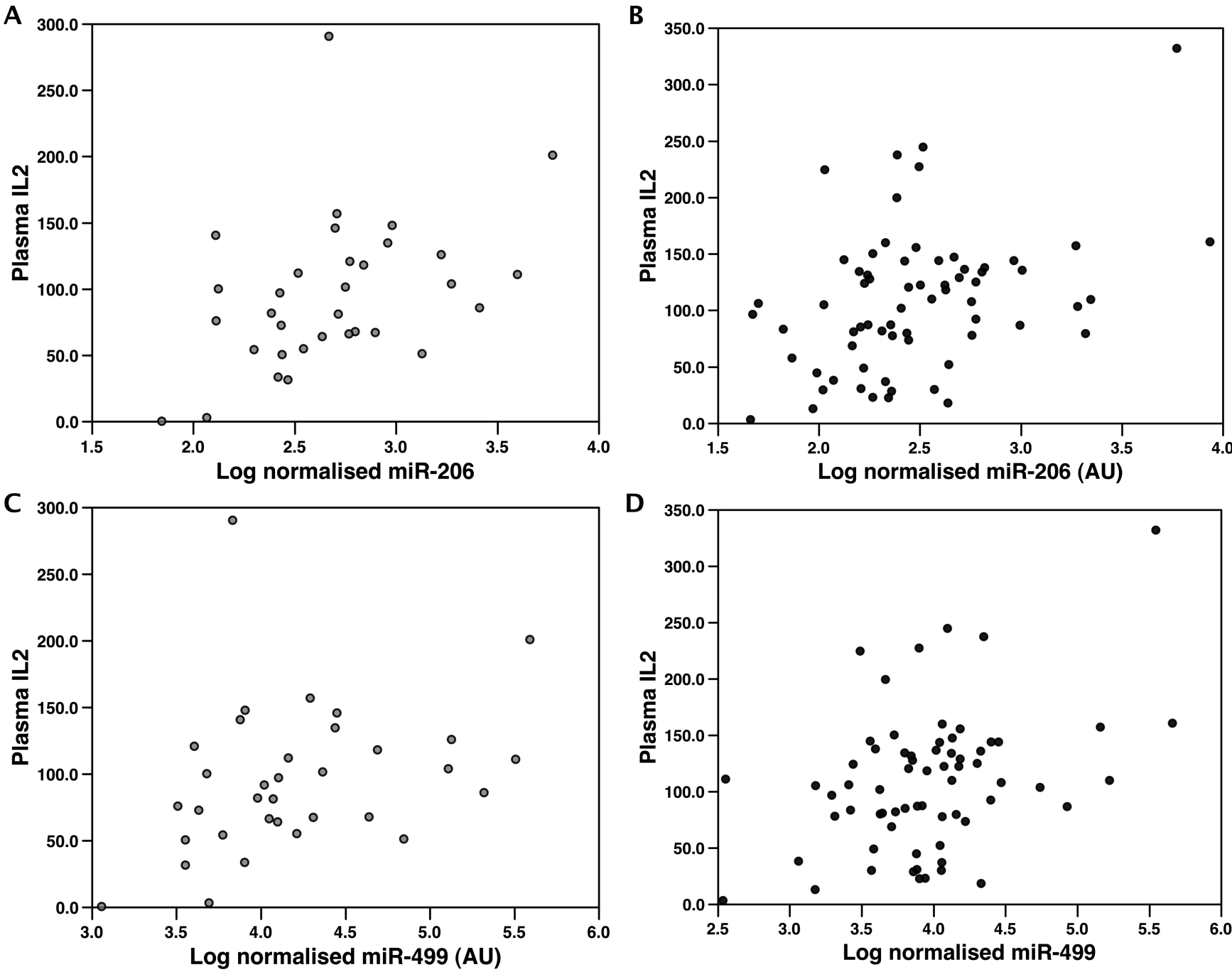

Figure 7 Plasma miR-499 and miR-206 are associated with plasma interleukin 2 (IL-2) in patients with chronic obstructive pulmonary disease (COPD). Log normalised plasma miR levels (determined as described in 'Methods') were compared with plasma IL-2 levels determined by ELISA. miR-206 was not associated with plasma IL-2 in patients with Global Initiative for Obstructive Lung Disease (GOLD) I and II COPD but was positively associated with plasma IL-2 in GOLD III and IV COPD (B) $(r=0.39, p<0.001)$. miR-499 was not associated with IL-2 in patients with GOLD I and II COPD (C) or in patients with GOLD III and IV COPD (D). Patients with GOLD I and II COPD are represented by filled grey circles and those with GOLD III and IV COPD are represented by filled black circles.

stage, ${ }^{37}$ neither of which were seen. It is therefore likely that quadriceps muscle is the main contributor to changes in plasma myomiRs.
This study is a cross sectional analysis of a large cohort of patients with COPD with plasma myomiRs measured at a single time point and as such we can only speculate on changes that

Table 3 Pearson correlation coefficients for the association of plasma myomiRs with plasma cytokines

\begin{tabular}{|c|c|c|c|c|c|c|c|}
\hline \multirow[b]{2}{*}{ Patient group } & \multirow[b]{2}{*}{ Cytokine } & \multicolumn{2}{|c|}{ miR-499 } & \multicolumn{2}{|c|}{$\operatorname{miR}-133$} & \multicolumn{2}{|c|}{ Mir-206 } \\
\hline & & $r$ & $p$ & $r$ & $p$ & $r$ & $p$ \\
\hline & IL-2 & 0.33 & 0.056 & 0.35 & 0.049 & 0.32 & 0.07 \\
\hline \multirow[t]{3}{*}{ GOLD I/II } & IL-5 & 0.22 & 0.202 & 0.23 & 0.201 & 0.42 & 0.016 \\
\hline & TNF & 0.25 & 0.162 & 0.28 & 0.118 & 0.36 & 0.038 \\
\hline & IL-2 & 0.15 & 0.223 & 0.37 & 0.002 & 0.39 & $<0.001$ \\
\hline \multirow[t]{3}{*}{ GOLD III/IV } & IL-5 & 0.238 & 0.052 & 0.39 & $<0.001$ & 0.38 & $<0.001$ \\
\hline & TNF & 0.27 & 0.023 & 0.25 & 0.042 & 0.36 & 0.038 \\
\hline & IL-2 & 0.33 & $<0.001$ & 0.34 & $<0.001$ & 0.36 & $<0.001$ \\
\hline \multirow[t]{2}{*}{ All } & IL-5 & 0.31 & 0.002 & 0.32 & $<0.001$ & 0.34 & $<0.001$ \\
\hline & TNF & 0.22 & 0.026 & 0.22 & 0.025 & 0.27 & 0.007 \\
\hline
\end{tabular}

GOLD, Global Initiative for Obstructive Lung Disease; IL, interleukin; TNF, tumour necrosis factor.

Bold signifies statistically significant correlation $\mathrm{p}<0.01$. 
might occur over time. Our data are therefore correlative in nature and the associations do not show causation.

\section{CONCLUSIONS}

This study demonstrates elevated muscle-specific miRNAs in the plasma of patients with COPD compared with controls. Our findings are consistent with an increase in muscle wasting and turnover, associated with the development of skeletal muscle dysfunction and fibre-type shift in patients with COPD. Despite the complex pathophysiology underlying circulating myomiRs, the, albeit weak, correlations of plasma myomiRs with quadriceps function and fibre characteristics suggest that the quadriceps are a significant contributor to the changes in plasma myomiRs in COPD. We do not offer a biological function for the circulating miRs, but suggest that the amount reflects the underlying skeletal muscle mass, rate of turnover and muscle fibre-type composition of patients.

Contributors Conception of study: PK, MP. Generation of samples and carrying out experimental procedures: AD, SN, AL. Analysis of data: PK, AD, AL, SN, MP. Writing of first draft: AD, PK, AL. Critical appraisal of manuscript: all authors. MP and PK contributed equally

Funding This project was supported by the NIHR Respiratory Disease Biomedical Research Unit at the Royal Brompton and Harefield NHS Foundation Trust and Imperial College London, and funding from COPD-MAP. MP's salary is part funded and AD's wholly funded by the NIHR Respiratory Disease Biomedical Research Unit at the Royal Brompton and Harefield NHS Foundation Trust and Imperial College London. AL was part funded by the BBSRC BB/H530703/1 and part by the MRC through COPD-MAP G1001362.

Competing interests None.

Ethics approval Royal Brompton \& Harefield NHS Trust Research Ethics Committee (studies 06/Q0404/35 and 06/Q0410/54).

Provenance and peer review Not commissioned; externally peer reviewed.

Open Access This is an Open Access article distributed in accordance with the Creative Commons Attribution Non Commercial (CC BY-NC 3.0) license, which permits others to distribute, remix, adapt, build upon this work non-commercially, and license their derivative works on different terms, provided the original work is properly cited and the use is non-commercial. See: http://creativecommons.org/licenses/by-nc/3.0/

\section{REFERENCES}

1 Bernard S LBP, Whittom F, Carrier G, et al. Peripheral muscle weakness in chronic obstructive pulmonary disease. Am J Respir Crit Care Med 1998;158:629-34.

2 Gosker HR, Engelen MP, van Mameren $\mathrm{H}$, et al. Muscle fiber type IIX atrophy is involved in the loss of fat-free mass in chronic obstructive pulmonary disease. Am $\mathrm{J}$ Clin Nutr 2002;76:113-19.

3 Natanek SA, Gosker HR, Slot IG, et al. Heterogeneity of quadriceps muscle phenotype in chronic obstructive pulmonary disease (COPD); implications for stratified medicine? Muscle Nerve 2013; Published Online First: 30 January 2013 doi:10.1002/mus.23784

4 Gosker HR, van Mameren H, van Dijk PJ, et al. Skeletal muscle fibre-type shifting and metabolic profile in patients with chronic obstructive pulmonary disease. Eur Respir J 2002; 19:617-25

5 Kelly JL, Elkin SL, Fluxman J, et al. Breathlessness and skeletal muscle weakness in patients undergoing lung health screening in primary care. COPD;10:40-54.

6 Seymour JM, Spruit MA, Hopkinson NS, et al. The prevalence of quadriceps weakness in COPD and the relationship with disease severity. Eur Respir J 2010;36:81-8.

7 Shrikrishna D, Patel M, Tanner RJ, et al. Quadriceps wasting and physical inactivity in patients with COPD. Eur Respir J 2012

8 Swallow EB, Reyes D, Hopkinson NS, et al. Quadriceps strength predicts mortality in patients with moderate to severe chronic obstructive pulmonary disease. Thorax 2007;62:115-20.

9 Jakobsson P, Jorfeldt L, Brundin A. Skeletal muscle metabolites and fibre types in patients with advanced chronic obstructive pulmonary disease (COPD), with and without chronic respiratory failure. Eur Respir J 1990;3:192-6.

10 Whittom F, Jobin J, Simard PM, et al. Histochemical and morphological characteristics of the vastus lateralis muscle in patients with chronic obstructive pulmonary disease. Med Sci Sports Exerc 1998;30:1467-74.
11 Maltais F, Sullivan MJ, LeBlanc $P$, et al. Altered expression of myosin heavy chain in the vastus lateralis muscle in patients with COPD. Eur Respir J 1999; 13:850-4.

12 Satta A, Migliori GB, Spanevello A, et al. Fibre types in skeletal muscles of chronic obstructive pulmonary disease patients related to respiratory function and exercise tolerance. Eur Respir J 1997;10:2853-60.

13 Gosker $\mathrm{H}$, Zeegers $\mathrm{M}$, Wouters $\mathrm{E}$, et al. Muscle fibre type shifting in the vastus lateralis of patients with COPD is associated with disease severity: a systematic review and meta-analysis. Thorax 2007:62:944-9.

14 Steiner MC, Roubenoff R, Tal-Singer RM, et al. Prospects for the development of effective pharmacotherapy targeted at the skeletal muscles in chronic obstructive pulmonary disease. A translational review. Thorax 2012;67:1102-9.

15 Chen JF, Mandel EM, Thomson JM, et al. The role of microRNA-1 and microRNA-133 in skeletal muscle proliferation and differentiation. Nat Genet 2006;38:228-33.

16 Guller I, Russell AP. MicroRNAs in skeletal muscle: their role and regulation in development, disease and function. J Physiol 2010;588(Pt 21):4075-87.

17 van Rooij E, Quiat $D$, Johnson BA, et al. A family of microRNAs encoded by myosin genes governs myosin expression and muscle performance. Dev Cell 2009; 17:662-73.

18 McCarthy JJ, Esser KA, Peterson CA, et al. Evidence of MyomiR network regulation of beta-myosin heavy chain gene expression during skeletal muscle atrophy. Physiol Genomics 2009;39:219-26.

19 Lewis A, Riddoch-Contreras J, Natanek SA, et al. Downregulation of the serum response factor/miR-1 axis in the quadriceps of patients with COPD. Thorax 2012;67:26-34

20 Mitchell PS, Parkin RK, Kroh EM, et al. Circulating microRNAs as stable blood-based markers for cancer detection. Proc Natl Acad Sci U S A 2008;105:10513-18.

21 Alevizos I, Illei GG. MicroRNAs as biomarkers in rheumatic diseases. Nat Rev Rheumatol 2010;6:391-8.

22 Rabe KF, Hurd S, Anzueto A, et al. Global strategy for the diagnosis, management, and prevention of chronic obstructive pulmonary disease: GOLD executive summary. Am J Respir Crit Care Med 2007;176:532-55.

23 Natanek SA, Riddoch-Contreras J, Marsh GS, et al. Yin Yang 1 expression and localisation in quadriceps muscle in COPD. Arch Bronconeumol 2011; 47:296-302.

24 Riddoch-Contreras J, George T, Natanek SA, et al. p38 Mitogen-activated protein kinase is not activated in the quadriceps of patients with stable chronic obstructive pulmonary disease. COPD 2012;9:142-50.

25 Bergström J. Muscle electrolytes in man. Determination by neutron activation analysis on needle biopsy specimens. A study on normal subjects, kidney patients and patients with chronic diarrhoea. Scand J Clin Lab Invest 1962;14(Suppl 68):1-110.

26 Ellis PD, Smith CW, Kemp P. Regulated tissue-specific alternative splicing of enhanced green fluorescent protein transgenes conferred by alpha-tropomyosin regulatory elements in transgenic mice. J Biol Chem 2004;279:36660-9.

27 Kroh EM, Parkin RK, Mitchell PS, et al. Analysis of circulating microRNA biomarkers in plasma and serum using quantitative reverse transcription-PCR (qRT-PCR). Methods 2010;50:298-301.

28 Chang J, Nicolas E, Marks D, et al. miR-122, a mammalian liver-specific microRNA, is processed from hor mRNA and may downregulate the high affinity cationic amino acid transporter CAT-1. RNA Biol 2004;1:106-13.

29 Cacchiarelli D, Legnini I, Martone J, et al. miRNAs as serum biomarkers for Duchenne muscular dystrophy. EMBO Mol Med 2011;3:258-65.

$30 \mathrm{Hu}$ Z, Chen X, Zhao Y, et al. Serum microRNA signatures identified in a genome-wide serum microRNA expression profiling predict survival of non-small-cell lung cancer. J Clin Oncol 2010;28:1721-6.

31 Hunter RB, Kandarian SC. Disruption of either the Nfkb1 or the $\mathrm{BCl} 3$ gene inhibits skeletal muscle atrophy. J Clin Invest 2004;114:1504-11.

32 Collino F, Deregibus MC, Bruno S, et al. Microvesicles derived from adult human bone marrow and tissue specific mesenchymal stem cells shuttle selected pattern of miRNAs. PLoS One 2010;5:e11803.

33 Kim HK, Lee YS, Sivaprasad U, et al. Muscle-specific microRNA miR-206 promotes muscle differentiation. J Cell Biol 2006;174:677-87.

34 Dey BK, Gagan J, Dutta A. miR-206 and -486 induce myoblast differentiation by downregulating Pax7. Mol Cell Biol 2011;31:203-14.

35 Fichtlscherer S, Zeiher AM, Dimmeler S. Circulating microRNAs: biomarkers or mediators of cardiovascular diseases? Arterioscler Thromb Vasc Biol 2011:31:2383-90.

36 Seymour JM, Ward K, Raffique A, et al. Quadriceps and ankle dorsiflexor strength in chronic obstructive pulmonary disease. Muscle Nerve 2012;46:548-54.

37 Stubbings AK, Moore AJ, Dusmet $\mathrm{M}$, et al. Physiological properties of human diaphragm muscle fibres and the effect of chronic obstructive pulmonary disease. J Physiol 2008;586:2637-50. 